\title{
Evaluation of the antimicrobial, antioxidant and physicochemical properties of Poly(Vinyl chloride) films containing quercetin and silver nanoparticles
}

\author{
Lilian R. Braga ${ }^{\mathrm{a}, *}$, Leonardo M. Pérez ${ }^{\mathrm{b}, \mathrm{c}}$, Marina del V. Soazo ${ }^{\mathrm{b}}$, Fabricio Machado ${ }^{\mathrm{a}, * *}$ \\ ${ }^{a}$ Laboratory of Chemical Processes Development, University of Brasília, Institute of Chemistry, Campus Universitário Darcy Ribeiro, CEP 70904-970, Brasília, DF, Brazil \\ ${ }^{\mathrm{b}}$ Facultad de Ciencias Bioquímicas y Farmacéuticas, Universidad Nacional de Rosario (UNR) \& Instituto de Química de Rosario (UNR-Consejo Nacional de Investigaciones \\ Científicas y Técnicas-CONICET), Suipacha 570, S2002LRL, Rosario, Santa Fe, Argentina \\ ${ }^{\mathrm{c}}$ Depto. de Investigación Institucional, Facultad de Química e Ingeniería del Rosario, Pontificia Universidad Católica Argentina (UCA), Av. Pellegrini 3314, S2002QEO, \\ Rosario, Santa Fe, Argentina
}

\section{A R T I C L E I N F O}

\section{Keywords:}

Poly(vinyl chloride) films

Quercetin

Silver nanoparticles

Antioxidant properties

Antimicrobial activity

\begin{abstract}
A B S T R A C T
This work provides details regarding the physicochemical, antimicrobial and antioxidant properties of poly(vinyl chloride) (PVC)-based films containing $0.4 \%$ quercetin and silver nanoparticles (AgNPs) at various concentrations levels. The incorporation of quercetin and AgNPs into the PVC matrix considerably affected the thermal, mechanical and optical properties of the films. Results obtained from the tensile stresgth test, demonstrated an improvement in the mechanical strength of the films after the incorporation of both quercetin and AgNPs. Moreover, an increase in AgNPs concentration increased the rigidity, as compared to control PVC film. Antimicrobial activity against food pathogens (Escherichia coli, Salmonella Typhimurium and Listeria monocytogenes) was evaluated by an antimicrobial barrier test. Results showed that the PVC-based films with quercetin and AgNPs proved to be highly effective to inhibiting bacterial growth. Therefore, these results indicate promising evidence to possibly aid in the prevention of microbial dissemination in foods. Additionally, films incorporated with quercetin and AgNPs expressed an antioxidant capacity when evaluated via the DPPH method. Among all of the films evaluated, the PVC-based films containing $0.4 \%$ quercetin and $1 \%$ AgNPs were flexible, exhibiting excellent UV-light barrier properties and for use with fatty foods, with the intent of reducing lipid oxidation and preventing food pathogen dissemination.
\end{abstract}

\section{Introduction}

In recent years, food technologists have been observing the development of new packaging tools that not only ensure food safety but also extend shelf life, while preserving the sensory characteristics of foods, such as appearance, consistency, texture, and flavor. In such sense, the development of active films that prevent lipid oxidation and microorganism growth and/or dissemination on foods is considered a very promising research field (Rhim, Wang, \& Hong, 2013).

Different types of materials are used in the production of these new packages, such as textile fibers, papers, and polymers (films/labels/ sachets), thus providing and opportunity for the incorporation or immobilization of active agents of their structure, surface in order to improve the final package performance, extending shelf life and/or enhance the quality of the food packaging (Boonnattakorn, Chonhenchob, Siddiq, \& Singh, 2015).
Furthermore, a key factor which may influence the processing and synthesis of active films is the type of polymer employed in the manufacture. Synthetic polymers present some advantages, such as low density, inert behavior, excellent barrier properties, high transparency, good mechanical strength, and relatively easy manipulation. With respect of these polymers, polypropylene (PP), low density polyethylene (LDPE), poly(ethylene terephthalate) (PET), poly(ethylene-co-vinyl alcohol) (EVOH), poly(ethylene-co-vinyl acetate) (EVA) and poly(vinyl chloride) (PVC) have drawn special attention, as they are the synthetic polymers most, frequently used for active packaging development (Sung et al., 2013). Among them, PVC is the second most widely used thermoplastic in the world due to its great versatility, non-toxic and inert quality that ensures its applicability in several areas, specifically the food industry (Cushen, Kerry, Morris, Cruz-Romero, \& Cummins, 2013). According to the Association of Plastics Manufacturers in Europe (PlasticsEurope, 2017) the world and European Union plastics

\footnotetext{
* Corresponding author.

*** Corresponding author.

E-mail addresses: lilianrodribraga@gmail.com (L.R. Braga), perez@inv.rosario-conicet.gov.ar (L.M. Pérez), marinasoazo@yahoo.com.ar (M.d.V. Soazo), fmachado@unb.br (F. Machado).
} 
production data, considering several plastic materials, such as thermoplastics, polyurethanes, thermosets, adhesives, coatings, and sealants, reveal that world demand for plastics resin was of 335 million tons in 2016. Noting that total demand of PVC at $39.9 \%$ was employed in the packaging industry.

As a matter of fact, antimicrobial and antioxidant agents play a fundamental role on the polymer-based films. In this scenario, nanomaterials possess unique chemical and physical properties that make them desirable to be incorporated in various polymer matrix for many consumer purposes. This has led to a major increase in the number of household products that incorporate nanomaterials. An inevitable consequence of this surge in use is that nanomaterials are increasingly prevalent in waste streams. To the best of our knowledge, only a few recycling and re-use strategies have been developed for nanoparticles (Deep et al., 2011). Designing these strategies is challenging because, reuse techniques must be practical, relatively simple, cheap, fast, and energy efficient. Several methods have been developed for the extraction, separation, and re-use of expensive nanoparticles from complex mixtures and different liquids media, including wastewater (Myakonkaya, Guibert, Eastoe, \& Grillo, 2010; Myakonkaya, Hu, Nazar, \& Eastoe, 2010; Pati, McGinnis, \& Vikesland, 2016).

Several antimicrobial and antioxidant agents have been proposed in active packaging fields to enhance the polymer film properties. Antimicrobials may include preferentially metal salts or metal nanoparticles (e.g., silver, cobalt, zinc), organic acids (e.g., sorbic acid, benzoic acid, propionic acid, acetic acid), natural essential oils (e.g., rosemary, thyme, oregano), bacteriocins (e.g., nisin and natamycin), and antimicrobial enzymes like lactoperoxidase and lactoferrin (Biji, Ravishankar, Mohan, \& Srinivasa Gopal, 2015). All these compounds are able to inhibit bacterial spoilage and pathogenic microorganism's growth in foods. Among them, active packaging including silver nanoparticles (AgNPs) stands out as the highest antimicrobial potential against a wide range of microorganisms including bacteria, fungi, and viruses (Gammariello, Conte, Buonocore, \& Del Nobile, 2011).

Moreover, antioxidant agents used in active packaging can be natural (quercetin, $\alpha$-tocopherol, carvacrol, catechol), or synthetic phenolic compounds (butylated hydroxytoluene), organic acids (e.g., ascorbic acid, citric acid), or enzymes (e.g., glucose oxidase) (Wang, Li, Guan, \& Zhang, 2016). Antioxidants are incorporated in packaging films because these compounds suppress oxidation reactions in sensitive food products (Castro López, De Dicastillo, Vilariño, \& Rodríguez, 2013). Lipid oxidation is determinant for shelf life, especially in foods with a high-fat content like, cooked ham, cheese, and sausages. Additionally, antioxidant incorporation can also stabilize the polymer matrix in order to protect the active films against thermo-oxidative degradation (Biji et al., 2015).

In recent years, and especially due to the consumer's demand, there has been an increasing interest in the incorporation of natural antioxidants in active packaging. In this sense, natural polyphenolic compounds are receiving considerable attention due to its high antioxidant activity. Quercetin is considered to be one of the most abundant flavonoids found in the plant kingdom, being a crucial component of tea, fruits, vegetables, and wine. This polyphenol has been the object of multidisciplinary studies due to its antioxidant, anti-inflammatory, anticarcinogenic, and antiviral properties; acting as a free-radical scavenger or metal ions chelating agent (Souza et al., 2015).

Therefore, incorporation of antioxidant and antimicrobial agents in synthetic polymer films have demonstrated good results in the reduction of lipid oxidation and control of microbial growth in foods. Besides, the addition of active agents in the polymeric matrix of films can influence the mechanical, thermal, optical and gas barrier properties of the packaging (Tian, Decker, \& Goddard, 2013).

In this work, PVC-based films were developed incorporated with quercetin as an antioxidant, consisting of different concentrations of AgNPs as antimicrobial agent has been developed by using a one-step casting method. Physicochemical, mechanical, thermal, optical, antioxidant and antimicrobial characteristics of the obtained films were evaluated to assess the films applicability to be used in active packaging. This work was developed considering the recognised and reported antimicrobial activity of these two compounds, as for instance, quercetin is a polyphenolic flavonoid with chemoprotective properties, including antioxidant and antibacterial properties, while silver nanoparticles exhibit antimicrobial activity.

\section{Materials and methods}

\subsection{Materials}

Poly(vinyl chloride) (PVC) was kindly donated by the Brazilian Company Braskem S.A. (with a mass-average molar mass of $130 \mathrm{kDa}$ ). Silver nitrate (Labsynth, Diadema, SP, Brazil), quercetin hydrate (Sigma-Aldrich, Darmstadt, Germany), trisodium citrate (Ecibra, Santo Amaro, SP, Brazil), tetrahydrofuran (THF) (Tedia, USA), and 2,2Diphenyl-1-picrylhydrazyl (DPPH) (Sigma-Aldrich, Darmstadt, Germany) were purchased from suppliers. Escherichia coli ATCC 25922, Salmonella Typhimurium ATCC 14028 and Listeria monocytogenes ATCC 19115 were donated by the Clinical Bacteriology area, from the Faculty of Biochemical and Pharmaceutical Science, National University of Rosario, Argentina.

\subsection{Formation of active films}

Active films were prepared by solvent casting, following the modified procedure previously reported elsewhere (Amar \& Parisi, 2013; Braga, Rangel, Suarez, \& Machado, 2017). Initially, $0.5 \mathrm{~g}$ of PVC was slowly dissolved under constant agitation using a magnetic stirrer (model Go-Stirrer MS-H-S Go-Lab) in $20 \mathrm{~mL}$ THF containing $200 \mu \mathrm{L}$ of industrial epoxidized soybean oil as a plasticizer and kept under boiling $\left(65^{\circ} \mathrm{C}\right)$ for $120 \mathrm{~s}$. Subsequently, the homogeneous solutions were mixed with $0.4 \%$ of quercetin and silver nitrate (AgNPs) (0.0, 1.0, 4.0 and $8.0 \%)$. After agitation, an increase in the viscosity and reduction of the volume to $17 \mathrm{~mL}$ of the solution was noticed. Next, the solution was poured onto flat-surface glass plates and kept at $25^{\circ} \mathrm{C}$ until complete solvent evaporation. After formation of the films, they were immersed into a trisodium citrate solution $(1 \mathrm{mM})$, for $10 \mathrm{~min}$ for the formation of the silver nanoparticles (AgNPs). In parallel, a control PVC film was also prepared.

\subsection{Characterization}

The characterization of active films was performed following parameters: thickness, moisture content, soluble solids, light transmission, film transparency, scanning electron microscopy, transmission electronic microscopy, color analysis, FTIR-ATR analysis, and mechanical properties. Below is presented description for the most important parameters of this study. The description of the others being presented in the supplementary material.

\subsubsection{Antimicrobial barrier assay of PVC-based films in agar media}

In order to evaluate the film's performance in preventing microbial superficial contamination of foods, a microplate antimicrobial barrier assay was used according to the methodology proposed by Pérez, Soazo, Balagué, Rubiolo, and Verdini (2014). Five hundred microliters $(500 \mu \mathrm{L})$ of trypticase soy agar (TSA, pH 5.5) with the addition of $50 \mathrm{mg} / \mathrm{mL}$ of 2,3,5-triphenyl tetrazolium chloride (TTC; Merck, Darmstadt, Germany) was poured into 24-well microtiter plates (Greiner BioOne, Frickenhausen, Germany). The acidic condition of the media ( $\mathrm{pH}$ 5.5) was selected to provide a $\mathrm{pH}$ value similar to those reported for several foods, like cheeses and meats (Han \& Floros, 1997). Bacterial suspension $\left(\sim 5 \times 10^{3} \mathrm{CFU} / \mathrm{mL}\right)$ of Escherichia coli ATCC 25922, Salmonella Typhimurium ATCC 14028 or Listeria monocytogenes ATCC 19115 were prepared in saline solution $(\mathrm{NaCl} 0.85 \% \mathrm{wt} / \mathrm{v})$ from 
overnight cultures of each bacterial strain and were then used for inoculation for the microbial barrier test $16 \mathrm{~mm}$ diameter PVC-based films discs were cut from and applied to the surface of the wells filled with the agar medium. Then, $10 \mu \mathrm{L}$ of each bacterial inoculum were seeded on the film discs. Microplates were incubated at $37^{\circ} \mathrm{C}$ for $24 \mathrm{~h}$ until red colored colonies were observed by the naked eye. Experiments were carried out in triplicate.

\subsubsection{Antioxidant activity}

The efficacy of the films to scavenge 2,2'-diphenyl-1-picrylhydrazyl (DPPH) radicals was evaluated using the methodology described by Siripatrawan and Harte (2010), however, with some slight modifications. Briefly, the films were cut in $2 \mathrm{~cm}^{2}$ pieces, placed in test tubes and was $4 \mathrm{~mL}$ of a methanolic solution of DPPH $(0.06 \mathrm{mM})$ added. The mixture was shaken and then incubated in the dark at room temperature for $15 \mathrm{~min}$. Finally, reduction of DPPH absorbance was spectrophotometrically monitored at $515 \mathrm{~nm}$ for $0.25,1,2,5$ and $24 \mathrm{~h}$. The percentage of radical scavenging activity $(A c)$ was calculated according to the following equation:

$A c(\%)=\left(\frac{A b s_{D P P H-} A b s_{\text {sample }}}{A b s_{D P P H}}\right) \times 100$

Where blank was methanol, $A b s_{D P P H}$ and $A b s_{\text {sample }}$ are the absorbance of DPPH initial and sample, respectively. Results were expressed in $\mu \mathrm{g}$ Trolox equivalent/g film, as the average of three replicates.

\section{Results and discussion}

\subsection{Thickness, soluble solids, moisture content}

The values obtained for the thickness, moisture content (MC) and solubility in water of PVC-based films incorporated with quercetin and AgNPs in different concentrations are presented in Table 1.

The incorporation of quercetin in the polymeric matrix of PVC did not exhibit significant statistical difference $(p<0.05)$ of thickness compared to control film. Results reported by Han, Lu, and Ge (2014) did not show changes in the thickness with the incorporation of $0.74 \%$ and $1.23 \%$ of quercetin into high-density polyethylene (HDPE) and ethylene-vinyl acetate copolymer (EVA) films.

On the other hand, with the addition of quercetin and AgNPs (Quercetin + AgNPs) resulted in a significant increase in the thickness of the film compared to PVC and PVC-Quercetin films. In this case, the AgNPs acted as mechanical thixotropic additives that can adhere or interlock in the regular polymeric structure of the PVC-based films. In addition, the increased viscosity was observed for the film forming solutions with the addition of quercetin and AgNPs, which may result in thicker films (Taylor, Taylor, Dutton, \& De Kock, 2005).

The incorporation of quercetin and AgNPs into the polymeric matrix did not alter the integrity nor the malleability of the films after the 24-h immersion in water. Water solubility of all films developed in the present study was also low. The minimum increase in soluble solids

\section{Table 1}

Values (average \pm standard) of thickness $(n=10)$, soluble solids and moisture content $(n=3)$ of PVC-based incorporated with quercetin and AgNPs.

\begin{tabular}{llll}
\hline Film & Thickness $(\mu \mathrm{m})$ & Soluble solids (\%) & $\begin{array}{l}\text { Moisture content } \\
(\%)\end{array}$ \\
\hline PVC & $36.0 \pm 3.4^{\mathrm{a}}$ & $0.7 \pm 0.5^{\mathrm{a}}$ & $\mathrm{a}$ \\
PVC-Q & $36.8 \pm 1.8^{\mathrm{a}}$ & $0.9 \pm 0.1^{\mathrm{a}}$ & $\mathrm{a}$ \\
PVC-Q + AgNP 1\% & $41.2 \pm 1.8^{\mathrm{b}}$ & $1.1 \pm 0.1^{\mathrm{a}}$ & $\mathrm{a}$ \\
PVC-Q + AgNP 4\% & $45.9 \pm 1.9^{\mathrm{c}}$ & $1.0 \pm 0.5^{\mathrm{a}}$ & $2.4 \pm 0.1^{\mathrm{a}}$ \\
PVC-Q + AgNP 8\% & $45.8 \pm 2.9^{\mathrm{c}}$ & $2.1 \pm 0.1^{\mathrm{b}}$ & $2.5 \pm 0.6^{\mathrm{a}}$ \\
\hline
\end{tabular}

Values with different letters in each column are significantly different $(p<0.05)$.

${ }^{\text {a }}$ ND, non-detected. observed for PVC-Quercetin films incorporated with AgNPs could be attributed to a marginal loss of silver nanoparticles from films that may be dispersed in the liquid medium because of the strong agitation during the solubility assay. The moisture content of PVC-based films was low and not significantly different, which could be attributed to the uniform storage conditions applied. These results are attributed to the hydrophobic nature of PVC, quercetin, and AgNPs (Suganya \& Shanmugavelayutham, 2014). However, a marginal moisture content value was calculated for PVC-Quercetin films incorporated with $4 \%$ and 8\% AgNPs. This behavior may be associated with the hygroscopic nature due to an increasing number of citrates, stabilizing nanoparticles in the bioactive films.

Low water solubility and moisture content of films are desirable features in order to increase film durability and integrity when used as packaging for foods. In addition, the film water solubility can enhance its use for protection of the packaged product from the external environment. The film may be required to be water-insoluble, especially when applied in high moisture foods.

\subsection{Light transmission and film transparency}

UV-light transmission ( $\mathrm{T}$ ) and transparency $\left(\mathrm{T}_{600}\right)$ of PVC-based films are presented in Table 2. As observed, PVC control films showed light transmission values in the UV region ranging from $53.77 \pm 11.45 \%$ to $74.90 \pm 7.17 \%$, higher than those observed for PVC-Quercetin and PVC/quercetin films incorporated with AgNPs. These results show that PVC films present modest barrier properties to UV light due to the amorphous structure of the polymer matrix that provides a transparency to the films (Amar \& Parisi, 2013; Braga et al., 2017).

The UV-light barrier feature of the PVC-based films was significantly improved by the presence of both quercetin and AgNPs (Table 2). Quercetin is a polyphenolic compound from the flavonoid group, so the phenolic and pyran rings in the quercetin structure determine the high absorbance observed for this organic molecule in the UV region. (Mishurov, Voronkin, Roshal, \& Bogatyrenko, 2017).

Moreover, the addition of AgNPs to the PVC-Quercetin films decreased UV-light transmittance, since the light scattering was triggered by the nanoparticles dispersed in the film matrix (Arfat, Ejaz, Jacob, \& Ahmed, 2017). In addition, the reduction of light transmission could also be preferred due to an increase in the thickness of the film (see Table 1).

Therefore, the PVC-based films with the incorporation of quercetin and AgNPs obtained at the present study presented optical properties (UV-light barrier and transparency) suitable for their use in the food packaging industry.

\subsection{Color analysis}

The visual aspect of a film considered for food packaging applications is a very important attribute that may influence consumers' satisfactoriness. Furthermore, the total color difference $(\Delta \mathrm{E})$ is a good indicator of the color of a film because this value includes lightness (L), red-green hue (a), and yellow-blue shade (b) (Ramos et al., 2013).

As can be seen in Fig. 1, there are no changes in the value observed for $\triangle \mathrm{E}$ PVC and PVC-Quercetin films. Both films showed low $\Delta \mathrm{E}$ and looked fairly transparent to the naked eye, in spite of the yellowish color provided by quercetin that impacted in films transparency determined at $600 \mathrm{~nm}$ (Table 2). However, the incorporation of AgNPs induced a significant increment $(p<0.05)$ in that $\Delta \mathrm{E}$ was dependent on the nanoparticle's concentration. These changes are related with higher $\mathrm{b}^{*}$ and lower $\mathrm{L}^{*}$ values calculated for the PVC-based films with the addition of AgNPs (data not shown).

Our results are in agreement with those reported by Rhim et al. (2013), which expressed lower L-values and higher $\Delta \mathrm{E}$ for agar composite films incorporated with silver nanoparticles. At this point, it is 
Table 2

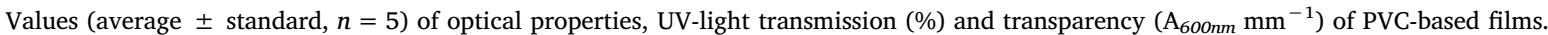

\begin{tabular}{|c|c|c|c|c|c|}
\hline \multirow[t]{2}{*}{ Films } & \multicolumn{5}{|l|}{ Wavelength (nm) } \\
\hline & 250 & 300 & 350 & 400 & $\mathrm{~T}_{600}$ \\
\hline PVC & $53.77 \pm 11.45^{\mathrm{a}}$ & $64.18 \pm 9.11^{\mathrm{a}}$ & $70.90 \pm 8.30^{\mathrm{a}}$ & $74.90 \pm 1.17^{\mathrm{a}}$ & $0.23 \pm 0.06^{\mathrm{a}}$ \\
\hline PVC-Q & $7.01 \pm 1.77^{\mathrm{b}}$ & $10.01 \pm 4.12^{\mathrm{b}}$ & $12.49 \pm 6.39^{\mathrm{b}}$ & $17.09 \pm 8.34^{\mathrm{b}}$ & $1.14 \pm 0.36^{\mathrm{b}}$ \\
\hline PVC-Q + AgNP 1\% & $4.53 \pm 0.12^{b c}$ & $5.55 \pm 0.37^{c}$ & $6.56 \pm 0.08^{\mathrm{b}}$ & $8.18 \pm 0.17^{c}$ & $2.38 \pm 0.09^{c}$ \\
\hline PVC-Q + AgNP 4\% & $3.72 \pm 0.57^{c}$ & $4.23 \pm 0.56^{\mathrm{cd}}$ & $5.04 \pm 0.31^{b}$ & $5.55 \pm 0.25^{c}$ & $2.61 \pm 0.07^{\mathrm{c}}$ \\
\hline PVC-Q + AgNP 8\% & $1.07 \pm 0.23^{\mathrm{d}}$ & $3.18 \pm 0.93^{\mathrm{d}}$ & $2.65 \pm 1.64^{\mathrm{c}}$ & $0.45 \pm 0.13^{\mathrm{d}}$ & $3.26 \pm 0.09^{d}$ \\
\hline
\end{tabular}

Values with different letters in each column are significantly different $(p<0.05)$.

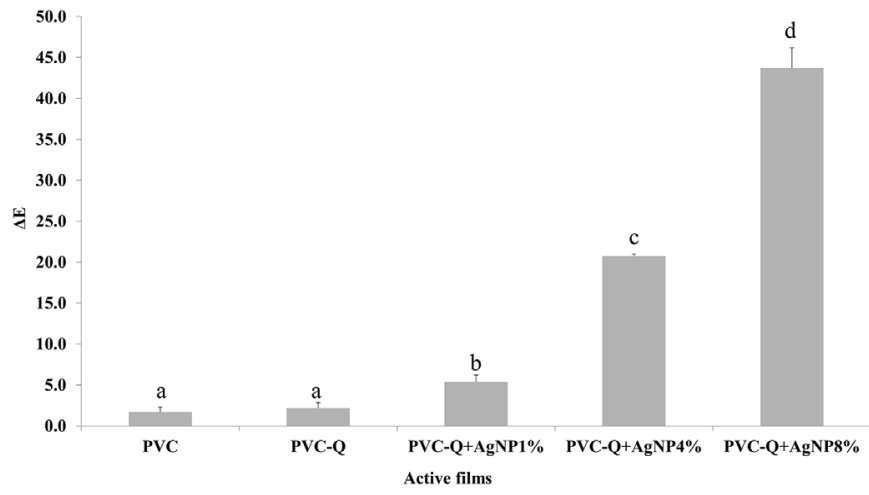

Fig. 1. $\Delta \mathrm{E}$ for active films values (average \pm standard, $n=5$ ). Different letters mean statistically significant differences $(p<0.05)$.

firmly stated that for practical uses, any color issue may be overcome with the addition of a coloring agent to the film formulation.

\subsection{Scanning electron microscopy}

Scanning electron microscopic (SEM) images of the surface of the PVC-based films are presented in Fig. 2. As observed, PVC films (Fig. 2A) present a surface with small pores and without a defined geometric shape (i.e., irregular morphology). On the other hand, the incorporation of quercetin into the polymeric matrix (Fig. 2B) revealed a regular network with a geometric formation on the surface of the film that exhibited pores ranging from $14.5 \mu \mathrm{m}$ to $20.3 \mu \mathrm{m}$ in diameter. The quercetin regular network seems to not be totally integrated into the PVC polymer matrix. This observation concurs with the establishment that a colloid mixture of isolated particles of quercetin and PVC that form a less cohesive film, as stated previously.

When AgNPs (1\%) was incorporated into PVC-Quercetin films, a decreased in the pore size of the regular network on the film surface was observed (Fig. 2C). This observation may be related to a decrease in the free quercetin concentration due to chemical interactions between this molecule and the AgNPs. The hydroxyl and oxo-groups exhibited in the quercetin structure have the facility to form complexes with various metal ions that can adhere or interlock into the regular polymeric structure of the PVC-based films (Bukhari, Memon, Mahroof-Tahir, \& Bhanger, 2009).

In addition, an increase in AgNPs (4\% and 8\%) fraction caused a significant change in PVC-Quercetin film surface morphology (Fig. 2D and E), respectively. These changes may probably be due to an excess of silver ions that favor the total complexation of quercetin and the incorporation of the nanoparticles in the film, with the associated increase in thickness (see Table 1 ).

TEM images of PVC-based films support this hypothesis since it was possible to verify that an increase in silver ions concentration produced larger particles. According to Figure (S1 A) in the supplementary material, it can be observed spherical silver nanoparticles with sizes varying between 5.10 and $24.91 \mathrm{~nm}$. These values are close to those reported by Khan et al. (2011) related to the synthesis of polyvinylpyrrolidone films with the silver nanoparticle. Fig. S1 A shows that the incorporation of AgNP1\% in the film reduced the particle size in the regular network and without agglomeration. It is also observed that with the increase of the concentration to AgNP4\% and AgNP8\%, Fig. S1 $\mathrm{B}$ and $\mathrm{C}$, respectively, provide the formation of agglomerates of silver nanoparticles.

\subsection{FTIR-ATR analysis}

FTIR is a technique used to obtain information about the chemical bonding in a material and to identify elementary constituents. The FTIR spectra of quercetin, the epoxidized soybean oil used as the plasticizer agent, and the PVC-based films developed in the present study are shown in Fig. $\mathrm{S} 2$ in the supplementary material. The quercetin spectrum (Figure S2 A) show a broad characteristic peak that covers the range $3000-3500 \mathrm{~cm}^{-1}$ assigned to the $\mathrm{OH}$ group. Another prominent peak is present at $1550-1650 \mathrm{~cm}^{-1}$ corresponding to o-quinone molecules. Moreover, FTIR spectrum of the epoxidized soybean oil (Figure S2 B) shows characteristics of absorption bands located at $2934 \mathrm{~cm}^{-1}$ and $2851 \mathrm{~cm}^{-1}$ that correspond asymmetric and symmetric stretching vibration of alkanes, respectively. The band situated at $823 \mathrm{~cm}^{-1}$ corresponds to the epoxy group (Bueno-Ferrer, Garrigós, \& Jiménez, 2010).

As observed from Fig. S2 C, FTIR spectrum of PVC showed the expected distinctive absorption bands: $3000-2700 \mathrm{~cm}^{-1}$ (the stretching of C-H), $1500-1400 \mathrm{~cm}^{-1}$ (the wagging of the $\mathrm{CH}_{2}$ groups), and 1331$1255 \mathrm{~cm}^{-1}$ (the stretching of the $\mathrm{C}-\mathrm{H}$ from $\mathrm{ClCH}$ groups) (Kayyarapu, Mohommad, and Chekuri, 2016; Turhan, Doğan, \& Alkan, 2010).

In general, the spectra of the PVC-based films incorporated with quercetin and AgNPs (Figure S2 D-G) were similar to that of the PVC film. However, it was noted that the amplitude of the peaks located at $2934 \mathrm{~cm}^{-1}, \quad 2851 \mathrm{~cm}^{-1}, \quad 1741 \mathrm{~cm}^{-1}, \quad 1430 \mathrm{~cm}^{-1}, \quad 1386 \mathrm{~cm}^{-1}$, $1333 \mathrm{~cm}^{-1}, 1254 \mathrm{~cm}^{-1}$, and $950 \mathrm{~cm}^{-1}$ decreased with the increased concentration of AgNPs in the film. In addition, a slight shift of the peaks located at $2934 \mathrm{~cm}^{-1}, 2851 \mathrm{~cm}^{-1}, 1741 \mathrm{~cm}^{-1}, 1430 \mathrm{~cm}^{-1}$ were observed. All these results support that nanocomposites films were successfully synthesized.

\subsection{Thermogravimetric analysis (TGA)}

TGA is a powerful tool for determining polymer thermal stability. This technique is based on the measurement of mass loss of a material as a function of temperature in a controlled atmosphere. Fig. 3 shows the thermogravimetric curves of PVC films with the addition of quercetin and AgNPs. Thermal stability is represented by the weight loss of the sample after heating over the temperature range $25-700^{\circ} \mathrm{C}$. As displayed below, the thermal degradation of all PVC-based films occurs through two significant degradation steps.

The weight loss curves showed degradation of samples above $260{ }^{\circ} \mathrm{C}$ in two steps. The major degradation in the temperature range $276{ }^{\circ} \mathrm{C}-363^{\circ} \mathrm{C}$ is attributed to dehydrochlorination of PVC, while the second step presents the total weight loss resulted from the degradation of the dehydrochlorinated residuals (Mallakpour \& Javadpour, 2015). 


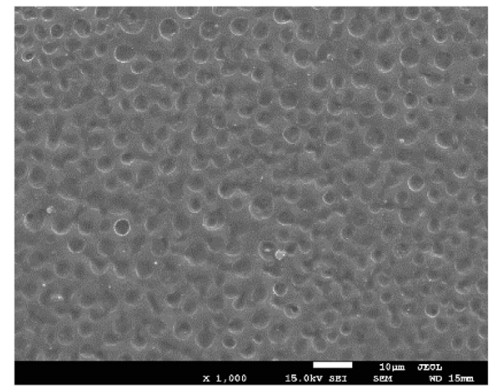

(A)

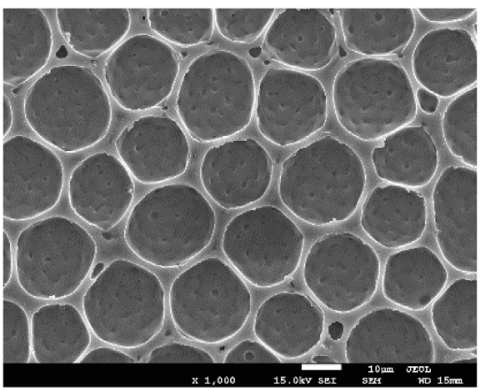

(B)

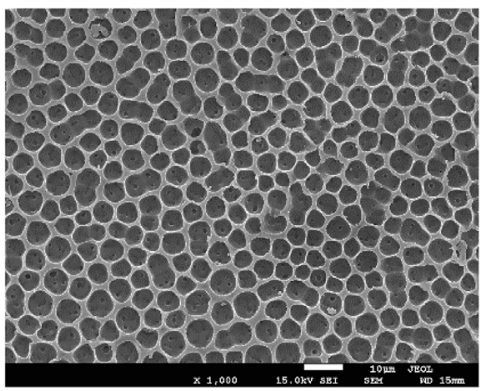

(C)

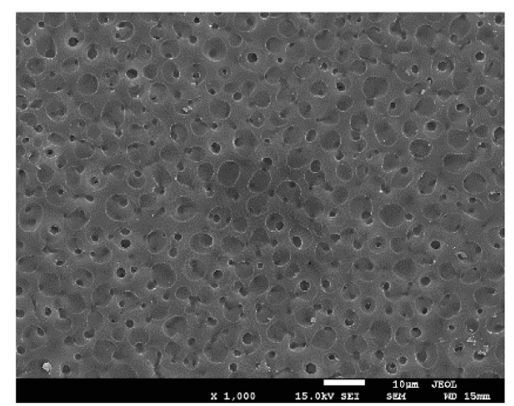

(D)

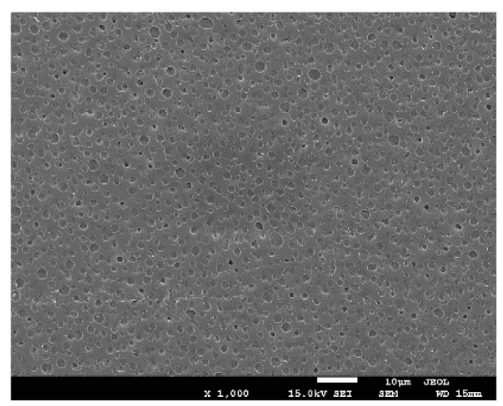

(E)

Fig. 2. SEM micrographs of the surface of the films with 1000 times magnification (15.0 KV) of active films. (A) poly(vinyl chloride) - PVC, (B) poly(vinyl chloride)/ quercetin - PVC-Q, (C) poly(vinyl chloride)/quercetin incorporated with silver nanoparticles $1.0 \mathrm{wt} \%$ - PVC-Q + AgNP1\%, (D) poly(vinyl chloride)/quercetin incorporated with silver nanoparticles $4.0 \mathrm{wt} \%-\mathrm{PVC}-\mathrm{Q}+\mathrm{AgNP} 4 \%$, and (E) poly(vinyl chloride)/quercetin incorporated with silver nanoparticles $8.0 \mathrm{wt} \%$ - PVC$\mathrm{Q}+\mathrm{AgNP} 8 \%$.

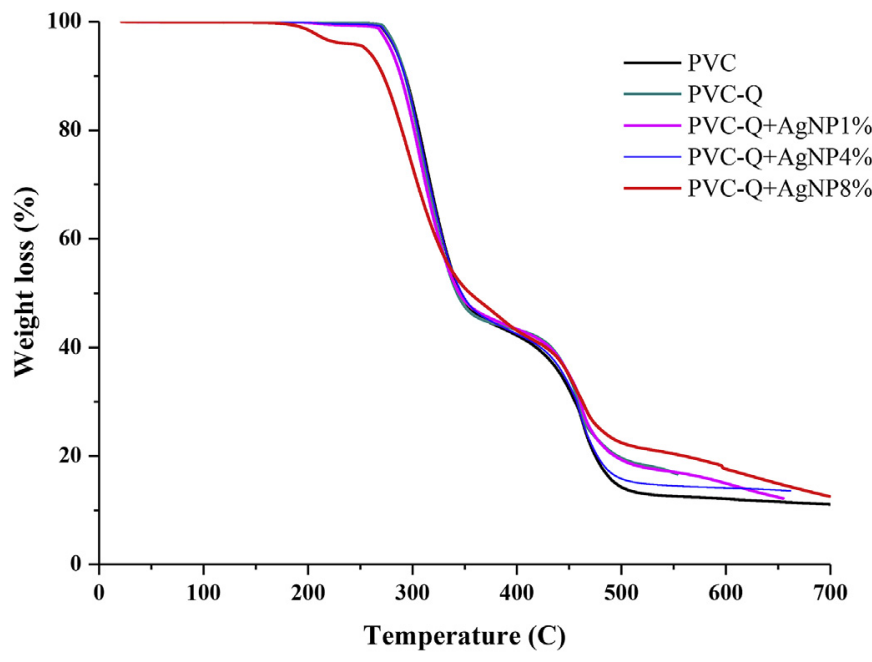

Fig. 3. Thermogravimetric analysis of based-PVC films. (A) poly(vinyl chloride) - PVC, (B) poly(vinyl chloride)/quercetin - PVC-Q, (C) poly(vinyl chloride)/ quercetin incorporated with silver nanoparticles $1.0 \mathrm{wt} \%$ - PVC-Q + AgNP1\%, (D) poly(vinyl chloride)/quercetin incorporated with silver nanoparticles $4.0 \mathrm{wt} \%$ - PVC-Q + AgNP4\%, and (E) poly(vinyl chloride)/quercetin incorporated with silver nanoparticles $8.0 \mathrm{wt} \%$ - PVC-Q + AgNP8\%.

Molecular reaction and radical propagation reactions become more probable and contribute to the cleavage of the $\mathrm{C}-\mathrm{Cl}$ bond. At a temperature higher than $363^{\circ} \mathrm{C}$, the chain radical mechanism becomes relevant and the melting of C-C or C-H bonds takes place (Kayyarapu, Mohommad, and Chekuri, 2016). The thermal degradation temperature of PVC-based films incorporating quercetin and AgNPs was shifted slightly to higher temperature according to pure PVC film. Probably, the presence of the organophilic silver nanoparticles strengthened the Van der Waals interactions between the PVC chains and improved the thermal stability of PVC, thus leading to an increased decomposition temperature (Turhan, Dog, and Alkan, 2010).

\subsection{Mechanical properties}

According to the transport and handling requirements of a food product, the desired properties of the film packaging will depend on the mechanical strength that is required to maintain the structural integrity and barrier properties of films. Besides, a suitable deformability property is also preferred for easy handling in most applications (Mali, Grossmann, García, Martino, \& Zaritzky, 2004).

Results corresponding to tensile strength (TS), elongation at break (EAB) and Young's Modulus (YM) of the PVC-based films incorporated with quercetin and AgNPs are shown in Table 3. PVC-Quercetin films showed significantly lower values of TS and YM than PVC film. The decrease of these TS values can be explained by the change in the intramolecular bonding by the addition of quercetin. The incorporation of

Table 3

Values (average \pm standard, $n=10$ ) of tensile strength (TS), elongation at break (EAB) and Young's Modulus (YM) of films.

\begin{tabular}{llll}
\hline Films & TS (Mpa) & EAB (\%) & YM (Mpa) \\
\hline PVC & $5.6 \pm 0.2^{\mathrm{b}}$ & $101 \pm 26^{\mathrm{b}}$ & $1.4 \pm 0.3^{\mathrm{b}}$ \\
PVC-Q & $2.6 \pm 0.2^{\mathrm{a}}$ & $203 \pm 22^{\mathrm{c}}$ & $0.7 \pm 0.1^{\mathrm{a}}$ \\
PVC-Q + AgNP 1\% & $9.3 \pm 0.4^{\mathrm{d}}$ & $94 \pm 36^{\mathrm{b}}$ & $1.4 \pm 0.4^{\mathrm{b}}$ \\
PVC-Q + AgNP 4\% & $8.3 \pm 0.5^{\mathrm{c}}$ & $71 \pm 36^{\mathrm{ab}}$ & $2.4 \pm 0.1^{\mathrm{c}}$ \\
PVC-Q + AgNP 8\% & $7.9 \pm 0.1^{\mathrm{c}}$ & $19 \pm 9^{\mathrm{a}}$ & $2.5 \pm 0.2^{\mathrm{c}}$ \\
\hline
\end{tabular}

Values with different letters in each column are significantly different $(p<0.05)$. 
a large polyphenolic molecule of the PVC-based films induces the structural development of discontinuities, resulting in a film structure with less chain mobility and, in consequence, less fracture resistance (Souza et al., 2015).

Hence, the incorporation of quercetin into the PVC matrix leads to a weaker and more flexible film, characterized by a lower mechanical resistance (TS), less stiffness (YM) and higher extensibility (EAB) in comparison with PVC films without theses additives. However, the incorporation of quercetin and AgNPs into PVC-based films significantly improved TS resulting in more resistant films. Similar observations were described by Lu, He, Wang, Lin, and Li (2014) regarding the production of antibacterial PVC films, with either silver-doped glass or silver-doped zirconium phosphate. These authors reported an increased in TS and YM in the doped films compared to the pure PVC ones, implying more rigid and less flexible films. Nevertheless, when the AgNPs concentration was changed from 1 to $8 \%$, the TS and EAB decreased. This behavior may be resultant to a rise in the nanoparticle complex size when increasing silver ions concentration, as stated previously. Therefore, larger aggregates of nanoparticles could interfere with the film backbone and generate a less cohesive internal structure, that certainly affects its mechanical properties.

\subsection{Antimicrobial barrier assay of active films}

A common food spoilage organism E. coli ATCC 25922 (a non-pathogenic surrogate of $E$. coli $\mathrm{O} 157: \mathrm{H7})$ and two pathogenic bacteria strains (S. Typhimurium ATCC 14028 and L. monocytogenes ATCC 19115) were employed to evaluate and prove the antimicrobial potential of the PVC-based films. Considering that post-processing surface bacterial contamination is a major issue for the food industry, film performance was assessed to prevent microbial contamination using a microplate antimicrobial barrier assay (Pérez et al., 2014). Results from the antimicrobial barrier test could simulate what would happen if bacteria post-contamination occurred on PVC film-wrapped or coated foods could be more appropriate in prospects for future applications in real food samples.

Bacterial growth was well inhibited, as observed in all PVC-based films for the three strains analyzed (Fig. 4). Although in PVC and PVCQuercetin films a complete growth inhibition was not reached, a marked reduction of the colonies number and size was clearly observed. These results strongly suggest that PVC-based films are highly innocuous to bacterial superficial contamination, mostly because of the low moisture content and water barrier properties of such films (Cushen et al., 2013). All of these aspects are crucial when a film is considered to be applied in food packaging since providing hygiene and food safety to the consumer.

\subsubsection{Antioxidant activity of active films}

The oxidation of food during storage causes a series of unfavorable changes in the sensory attributes of the product (e.g., rancidity, changes in color and texture) responsible for the quality decay and economic losses. Incorporation of antioxidants into food packaging materials to control the oxidation of fatty components and pigments can contribute to preservation of shelf life and food products quality (Souza et al., 2015).

The consumption of DPPH by PVC-based films combined with quercetin and AgNPs at different times are shown in Fig. 5. The ability of quercetin to stabilize free radicals was retained after its insertion into the PVC films. The high antioxidant activity observed in the PVCQuercetin film after $24 \mathrm{~h}(60.44 \pm 6.36 \%)$ may be attributed to the hydroxyl groups presented at the quercetin molecule and their low oxidation potential (Ravichandran, Rajendran, \& Devapiriam, 2014) that favors the rapid kidnap of DPPH free radicals in the solution. As expected, no antioxidant activity was observed for control PVC films.

The antioxidant activity of PVC-Quercetin + AgNPs films presented no significant statistical differences $(p<0.05)$ between them, reaching a maximum reduction of DPPH radicals at $24 \mathrm{~h}$ equivalent to $20.58 \pm 4.27 \%, 26.51 \pm 5.69 \%$ and $29.45 \pm 8.66 \%$ for $1 \%, 4 \%$ and $8 \%$ AgNPs, respectively. However, the formation of Quercetin + AgNPs complexes suggests that a reduction in the number of hydroxyl groups of quercetin is possible, and this behavior may be related to the decrease antioxidant activity observed for these films in comparison with the PVC-Quercetin films.

A similar behavior (i.e., lower antioxidant activity) was verified by (Ravichandran et al., 2014) when quercetin was complexed with cadmium ions. These results showed a potential for the use of PVC-based films to act as a carrier for quercetin, maintaining the antioxidant behavior of the free compound.

\section{Conclusion}

In this study, bioactive films based on PVC containing antioxidant

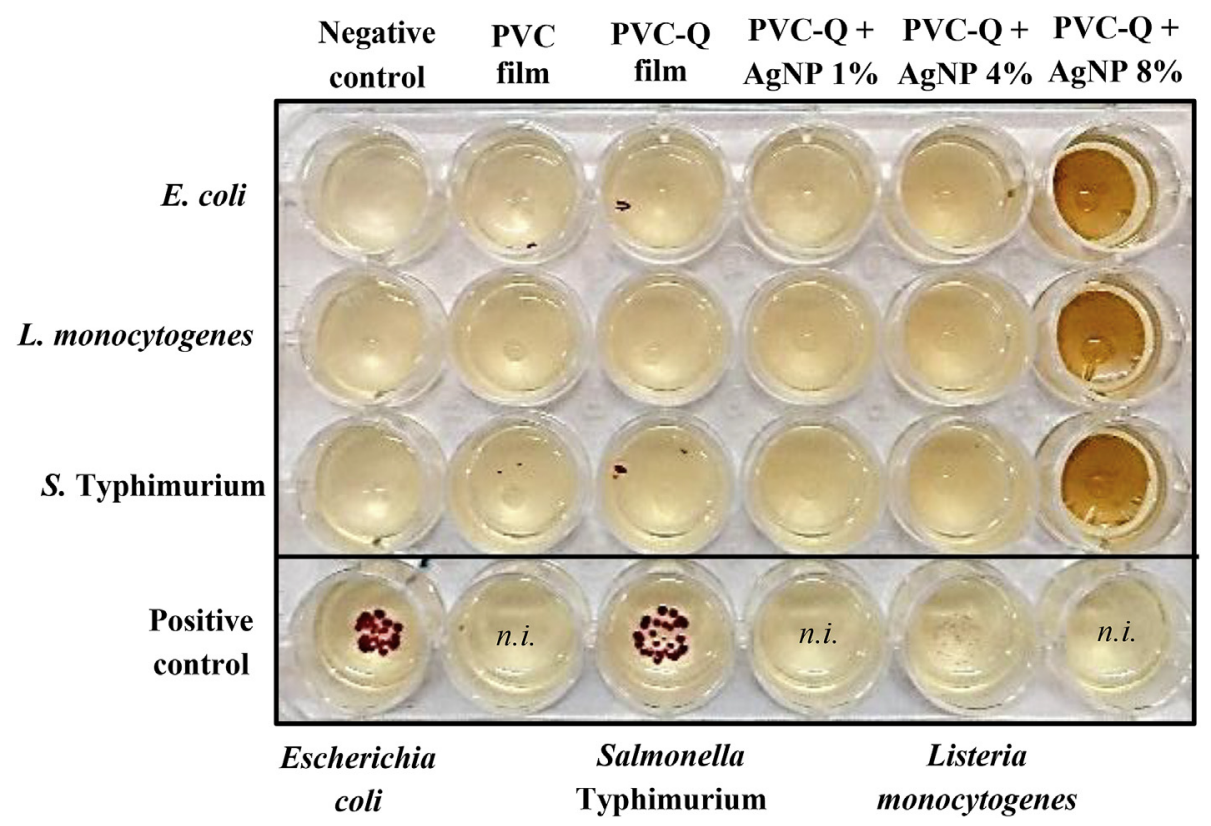

Fig. 4. Antimicrobial barrier assay of PVC-based films containing quercetin and quercetin with AgNPs. 


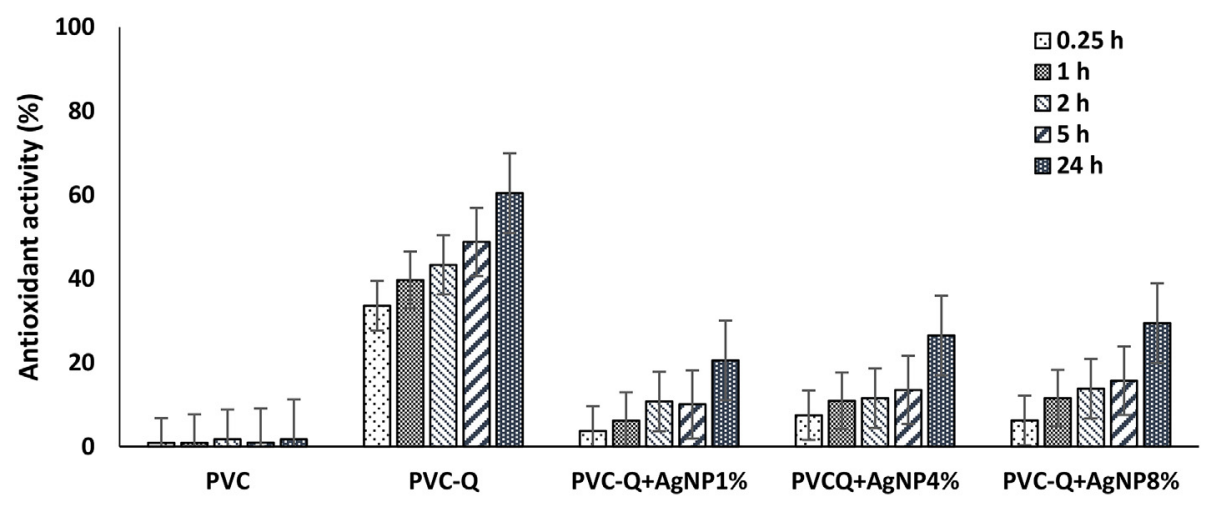

\section{Active Films}

Fig. 5. Percentage of antioxidant activities of active films containing quercetin and quercetin with AgNPs at different times. Different letters mean statistically significant differences $(p<0.05)$.

quercetin (Q) and antimicrobial silver nanoparticles (AgNPs) were successfully formulated, and synthetized via a simple and relatively fast, single step, casting method. The incorporation of both quercetin and AgNPs into the polymeric matrix of PVC influenced the film's optical, structural and mechanical properties. The PVC-based films formulated with quercetin and AgNPs presented an adequate transparency suitable for use in packaging, and prominent UV light barrier, and antioxidant properties. This is extremely appealing to the food industry as these features prevent lipid oxidation in fatty foods. Additionally, the tensile strength was increased and elongation at break was decreased when quercetin and AgNPs were incorporated in the PVC films. Moreover, the presence of quercetin and AgNPs enhanced the film's stability. In addition, all the PVC-based films showed strong antimicrobial activity against $E$. coli, $S$. Typhimurium and L. monocytogenes. These results suggested that PVC-based films containing quercetin and AgNPs have a promising potential to be used as a bioactive packaging material to improve food safety and quality.

\section{Acknowledgements}

The authors wish to acknowledge to FAPDF (Project no 193.001.433/2016) for its support and by fellowships, CNPq, CAPES and University of Brasília. We also want to thank Braskem S.A. for providing poly(vinyl chloride), Analytical Chemical Department of the Faculty of Biochemical and Pharmacological Sciences and Instituto de Química Rosario (IQUIR-CONICET, UNR) by the support in the realization of the laboratory tests.

\section{Appendix A. Supplementary data}

Supplementary data to this article can be found online at https:// doi.org/10.1016/j.lwt.2018.11.082.

\section{References}

Amar, A., \& Parisi, A. V. (2013). Spectral response of solvent-cast polyvinyl chloride (PVC) thin film used as a long-term UV dosimeter. Journal of Photochemistry and Photobiology B: Biology, 125, 115-120. https://doi.org/10.1016/j.jphotobiol.2013.05. 012.

Arfat, Y. A., Ejaz, M., Jacob, H., \& Ahmed, J. (2017). Deciphering the potential of guar gum/Ag-Cu nanocomposite films as an active food packaging material. Carbohydrate Polymers, 157, 65-71. https://doi.org/10.1016/j.carbpol.2016.09.069.

Biji, K. B., Ravishankar, C. N., Mohan, C. O., \& Srinivasa Gopal, T. K. (2015). Smart packaging systems for food applications: A review. Journal of Food Science \& Technology, 52, 6125-6135. https://doi.org/10.1007/s13197-015-1766-7.

Boonnattakorn, R., Chonhenchob, V., Siddiq, M., \& Singh, S. P. (2015). Controlled release of mangiferin using ethylene vinyl acetate matrix for antioxidant packaging. Packaging Technology and Science, 28, 241-252. https://doi.org/10.1002/pts.2097.

Braga, L. R., Rangel, E. T., Suarez, P. A. Z., \& Machado, F. (2017). Simple synthesis of active films based on PVC incorporated with silver nanoparticles: Evaluation of the thermal, structural and antimicrobial properties. Food Packaging and Shelf Life, 15, 122-129. https://doi.org/10.1016/j.fpsl.2017.12.005.

Bueno-Ferrer, C., Garrigós, M. C., \& Jiménez, A. (2010). Characterization and therma stability of poly(vinyl chloride) plasticized with epoxidized soybean oil for food packaging. Polymer Degradation and Stability, 95, 2207-2212. https://doi.org/10. 1016/j.polymdegradstab.2010.01.027.

Bukhari, S. B., Memon, S., Mahroof-Tahir, M., \& Bhanger, M. I. (2009). Synthesis, characterization and antioxidant activity copper-quercetin complex. Spectrochimica Acta Part A: Molecular and Biomolecular Spectroscopy, 71(5), 1901-1906. https://doi.org/ 10.1016/j.saa.2008.07.030.

Castro López, M. D. M., De Dicastillo, C. L., Vilariño, J. M. L., \& Rodríguez, M. V. G. (2013). Improving the capacity of polypropylene to be used in antioxidant active films: Incorporation of plasticizer and natural antioxidants. Journal of Agricultural and Food Chemistry, 61(35), 8462-8470. https://doi.org/10.1021/jf402670a.

Cushen, M., Kerry, J., Morris, M., Cruz-Romero, M., \& Cummins, E. (2013). Migration and exposure assessment of silver from a PVC nanocomposite. Food Chemistry, 139(1-4), 389-397. https://doi.org/10.1016/j.foodchem.2013.01.045.

Deep, A., Kumar, K., Kumar, P., Kumar, P., Sharma, A. L., Gupta, B., et al. (2011). Recovery of pure $\mathrm{ZnO}$ nanoparticles from spent $\mathrm{Zn}-\mathrm{MnO}_{2}$ alkaline batteries. Environmental Science \& Technology, 45(24), 10551-10556. https://pubs.acs.org/doi/ abs/10.1021/es201744t.

Gammariello, D., Conte, a, Buonocore, G. G., \& Del Nobile, M. a. (2011). Bio-based nanocomposite coating to preserve quality of Fior di latte cheese. Journal of Dairy Science, 94(11), 5298-5304. https://doi.org/10.3168/jds.2011-4161.

Han, J. H., \& Floros, J. D. (1997). Casting antimicrobial packaging films and measuring their physical properties and antimicrobial activity. Journal of Plastic Film and Sheeting, 13(4), 287-298. https://doi.org/10.1177/875608799701300405.

Han, T.-T., Lu, L.-X.,L., \& Ge, Changfeng. (2014). Development and properties of high density polyethylene (HDPE) and ethylene-Vinyl acetate copolymer (EVA) blend antioxidant active packaging films containing quercetin. Packaging Technology and Science, 28, 415-423.

Kayyarapu, B., Y, M. K., Mohommad, H. B., O, G. N., \& Chekuri, R. (2016). Structural, thermal and optical properties of pure and $\mathrm{Mn}^{2+}$ doped poly(vinyl chloride) films. Materials Research, 19(5), 1167-1175. https://doi.org/10.1590/1980-5373-MR2016-0239.

Khan, M. A. M., Kumar, S., Ahamed, M., Alrokayan, A., Alsalhi, M. S., Alhoshan, M., et al. (2011). Structural and spectroscopic studies of thin film of silver nanoparticles. Applied Surface Science, 257, 10607-10612. https://doi:10.1016/j.apsusc.2011.07. 058.

Lu, J.-Y., He, C., Wang, S.-Y., Lin, Z.-F., \& Li, W.-F. (2014). Preparation and properties of antibacterial polyvinylchloride. European Review for Medical for Pharmacological Science, 18(10), 1448-1453.

Mali, S., Grossmann, M. V. E., García, M. A., Martino, M. N., \& Zaritzky, N. E. (2004) Barrier, mechanical and optical properties of yam starch films. Carbohydrate Polymers, 56, 129-135. https://doi:10.1016/j.carbpol.2004.01.004.

Mallakpour, S., \& Javadpour, M. (2015). Design and characterization of novel poly(vinyl chloride) nanocomposite films with zinc oxide immobilized with biocompatible citric acid. Colloid \& Polymer Science, 293(9), 2565-2573. https://doi.org/10.1007/ s00396-015-3647-z.

Mishurov, D., Voronkin, A., Roshal, A., \& Bogatyrenko, S. (2017). Influence of structure $3,5,7,3^{\prime}, 4^{\prime}-$ Pentahydroxyflavone-based polymer films on their optical transparency. Optical Materials, 64, 166-170. https://doi.org/10.1016/j.optmat.2016.12.004.

Myakonkaya, O., Guibert, C., Eastoe, J., \& Grillo, I. (2010a). Recovery of nanoparticles made easy. Langmuir, 26(6), 3794-3797. https://pubs.acs.org/doi/abs/10.1021/ la100111b.

Myakonkaya, O., Hu, Z. Y., Nazar, M. F., \& Eastoe, J. (2010b). Recycling functional colloids and nanoparticles. Chemistry-a European Journal, 16(39), 11784-11790. https://doi.org/10.1002/chem.201000942.

Pati, P., McGinnis, S., \& Vikesland, P. J. (2016). Waste not want not: Life cycle implications of gold recovery and recycling from nanowaste. Environmental ScienceNano, 3(5), 1133-1143. https://doi.org/10.1039/C6EN00181E. 
Pérez, L. M., Soazo, M. del V., Balagué, C. E., Rubiolo, A. C., \& Verdini, R. A. (2014). Effect of $\mathrm{pH}$ on the effectiveness of whey protein/glycerol edible filmscontaining potassium sorbate to control non-O157 shiga toxin-producing Escherichia coli in ready-to-eat foods. Food Control, 37(1), 298-304. https://doi.org/10.1016/j. foodcont.2013.09.018.

PlasticsEurope Association of Plastics Manufacturers in Europe. (2017). https://www. plasticseurope.org/application/files/5715/1717/4180/Plastics_the_facts_2017 FINAL_for_website_one_page.pdf/Accessed 01 September 2017.

Ramos, Ó. L., Reinas, I., Silva, S. I., Fernandes, J. C., Cerqueira, M. A., Pereira, R. N., et al. (2013). Effect of whey protein purity and glycerol content upon physical properties of edible films manufactured therefrom. Food Hydrocolloids, 30(1), 110-122. https:// doi.org/10.1016/j.foodhyd.2012.05.001.

Ravichandran, R., Rajendran, M., \& Devapiriam, D. (2014). Antioxidant study of quercetin and their metal complex and determination of stability constant by spectrophotometry method. Food Chemistry, 146, 472-478. https://doi.org/10.1016/j. foodchem.2013.09.080.

Rhim, J. W., Wang, L. F., \& Hong, S. I. (2013). Preparation and characterization of agar/ silver nanoparticles composite films with antimicrobial activity. Food Hydrocolloids, 33(2), 327-335. https://doi.org/10.1016/j.foodhyd.2013.04.002.

Siripatrawan, U., \& Harte, B. R. (2010). Physical properties and antioxidant activity of an active film from chitosan incorporated with green tea extract. Food Hydrocolloids, 24(8), 770-775. https://doi.org/10.1016/j.foodhyd.2010.04.003.
Souza, M. P., Vaz, A. F. M., Silva, H. D., Cerqueira, M. A., Vicente, A. A., \& Carneiro-daCunha, M. G. (2015). Development and characterization of an active chitosan-based film containing quercetin. Food and Bioprocess Technology, 8(11), 2183-2191. https:// doi.org/10.1007/s11947-015-1580-2.

Suganya, A., \& Shanmugavelayutham, G. (2014). Analysis of adhesive and surface properties on poly vinyl chloride ( PVC ) treated by Dc. Glow Discharge Plasma, (August), 20-21.

Sung, S.-Y., Sin, L. T., Tee, T.-T., Bee, S.-T., Rahmat, A. R., Rahman, W. A. W. A., et al. (2013). Antimicrobial agents for food packaging applications. Trends in Food Science \& Technology, 33(2), 110-123. https://doi.org/10.1016/j.tifs.2013.08.001.

Taylor, J., Taylor, J. R. N., Dutton, M. F., \& De Kock, S. (2005). Identification of kafirin film casting solvents. Food Chemistry, 90(3), 401-408. https://doi.org/10.1016/j. foodchem.2004.03.055.

Tian, F., Decker, E. A., \& Goddard, J. M. (2013). Controlling lipid oxidation of food by active packaging technologies. Food \& Function, 4(5), 669. https://doi.org/10.1039/ c3fo30360h.

Turhan, Y., Doğan, M., \& Alkan, M. (2010). Poly(vinyl chloride)/kaolinite nanocomposites: Characterization and thermal and optical properties. Industrial \& Engineering Chemistry Research, 49(4), 1503-1513. https://doi.org/10.1021/ie901384x.

Wang, X., Li, Q., Guan, Y., \& Zhang, Y. (2016). Glucose oxidase-incorporated hydrogel thin film for fast optical glucose detecting under physiological conditions. Materials Today Chemistry, 1-2, 7-14. https://doi.org/10.1016/j.mtchem.2016.10.005. 